\title{
Myeloid Sarcoma Involving Kidneys: From Diagnosis to Treatment. Case Report and Literature Review
}

\begin{abstract}
Myeloid sarcomas (MS) are rare extramedullary hematological tumors which generally occur during the natural course of acute myeloid leukemia or chronic myeloid leukemia. Rarely, their onset precedes peripheral blood and bone marrow manifestations of disease. Common sites of involvement are skin, bone, soft tissue, lymph nodes, reproductive or digestive organs, and central nervous system.

Herein, we report the case of a 72-year-old man affected by JAK2 V617F mutated myeloproliferative neoplasm who developed MS involving collecting system of both kidneys. $M S$ and $M S$-related obstructive nephropathy were the first signs of the acute evolution of a known chronic hematological malignancy, preceding by some weeks the onset of leukocytosis.
\end{abstract}

Keywords: Sarcoma; Leukemia, Myeloid, Acute; Kidney

CMI 2020; 14(1): 39-44

http://dx.doi.org/10.7175/cmi.v14i1.1460

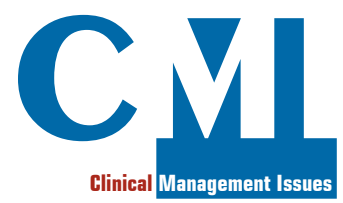

Case Report
Division of Hematology,

Department of

Medicine, Valduce

Hospital, Como, Italy,

2 Department of

Pathology, Valduce

Hospital, Como, Italy

\section{INTRODUCTION}

Myeloid sarcoma (MS), also known as "granulocytic sarcoma" or "chloroma", is a solid extramedullary tumor mass composed of malignant primitive myeloid cells. By definition, the infiltrates efface the underlying tissue architecture. In the 2016 revision to the World Health Organization classification of myeloid neoplasms and acute leukemia, MS is described as a unique clinical presentation of any subtype of acute myeloid leukemia (AML). The etiology of MS is still unknown. Myeloid sarcoma may present de novo, accompany peripheral blood and marrow involvement, present as relapse of acute myeloid leukemia, or as progression of a prior myelodysplastic syndrome (MDS), myeloproliferative neoplasm (MPN), or MDS/MPN. Seldom precedes MS the development of the hematologic disease [1].

Because of its rarity, MS is a diagnostic and therapeutic challenge. The differential diagnosis is with any atypical cellular infiltrate. MS may occur at any site, leading to very varied clinical presentations, but most commonly it affects lymph nodes, skin and soft tissues, bone, testes, gastrointestinal tract, and peritoneum. A high index of suspicion is required to diagnose MS, and

\section{Why Do We Describe This Case}

Myeloid sarcoma is a rare disease and standard diagnostic and therapeutic guidelines are lacking. It may involve many sites, thus making the diagnosis challenging, especially when its onset precedes the hematological disease or its evolution. In addition, the involvement of kidneys has been rarely described and may lead to important complications, thereby enhancing the importance of a timely diagnosis
Corresponding author Francesca Guidotti francesca_guidotti@yahoo.it

Received: 29 February 2020 Accepted: 5 May 2020 Published: 15 September 2020 
a combination of radiology, histology, immunophenotyping, and molecular analyses is essential for risk stratification and treatment planning.

MS may be categorized according with the degree of myeloid differentiation as:

- well-differentiated subtype;

- poorly differentiated subtype; and

- blastic subtype.

Immunohistochemistry shows CD68KP1 as the most commonly expressed marker, with a variable expression of myeloperoxidase, CD117, CD99, CD68/PG-M1, lysozyme, CD34, terminal deoxynucleotidyl transferase, CD56, CD61, CD30, glycophorin $\mathrm{A}$, and $\mathrm{CD} 4$.

MSs have several radiological features. Enhanced homogenous soft tissue masses at multiple sites strongly suggests the presence of an MS, especially when there is an underlying hematological disorder. Besides conventional radiologic investigations, positron emission/computed tomography (PET$\mathrm{CT}$ ) is a useful diagnostic tool in determining the site of MS [2].

Treatments for MS mainly include surgical resection, local radiotherapy, and systemic chemotherapy. Treatment response and median survival are not affected by age, gender, anatomical site, tissue type, phenotype, cytogenetics, and potential AML or myeloproliferative disease [3]. Surgical resection and local radiotherapy are not curative, since they do not delay the transformation of MS to AML or improve prognosis. However, these two approaches may be useful and indicated for symptomatic lesions or tumors causing local organ dysfunction or obstruction. Systemic chemotherapy using AML-like regimens is the first choice of treatment and should be started early, even in non-leukemic disease. Chemotherapy may be successful in terms of obtaining disease remission and can also prolong the survival period [4]. Allog- neic hematopoietic stem cell transplantation has demonstrated promising results, particularly in patients who achieved complete remission with AML-induction protocols.

The prognosis of MS is poor, with a short survival time. However, it largely varies among different studies. Al-Khateeb et al. reported a median survival of 24.7 months [3]. Li et al. described a mean survival time of 47.3 months, median survival time of 14 months, and the 1-, 2- and 5-year survival rates of $56.0 \%, 36.1 \%$, and $17.3 \%$ [5]. Early diagnosis and appropriate treatment strategies should be implemented before the disease progresses to AML, thereby ensuring better prognosis.

Herein, we describe a case of MS with unusual affected site, involving collecting system of both kidneys. The patient was affected by myeloproliferative neoplasm and MS onset preceded the classic leukemic manifestations. Diagnostic and therapeutic issues are discussed.

\section{CASE PRESENTATION}

In October 2016, a 72-year-old man was addressed to our Centre because of the detection at routine examination of splenomegaly and thrombocytosis. The patient had many comorbidities such as hypertension, type 2 diabetes, chronic renal failure, and chronic ischemic cardiopathy, which requested the placement of multiple medicated stents. Splenomegaly was confirmed by abdomen ultrasound together with thrombocytosis and leucocytosis, without other abnormalities observed in the complete blood count (Table I).

He was further investigated and a mutation of JAK2 V617F was detected. Bone marrow evaluation was not performed because of the hemorrhagic risk due to double anti-platelet therapy. The patient was diag-
Table I. Laboratory analyses performed at different timepoints.

$\mathrm{AML}=$ acute myeloid leukemia;

$\mathrm{MPN}=$ myeloproliferative neoplasm;

$\mathrm{WBC}=$ white blood cells

\begin{tabular}{|c|c|c|c|c|}
\hline Parameter & $\begin{array}{l}\text { Octoher } 2016 \\
\text { (MPN diagnosis) }\end{array}$ & $\begin{array}{c}\text { Fehruary } 2018 \\
\text { (admission } \\
\text { to hospital) }\end{array}$ & $\begin{array}{c}\text { March } 2018 \\
\text { (AML evolution) }\end{array}$ & Normal values \\
\hline Hemoglobin (g/dL) & 17.3 & 8.3 & 7.2 & $14-18$ \\
\hline WBC $\left(n \times 10^{9} / L\right)$ & 16.91 & 7.08 & 17.4 & $4-10.8$ \\
\hline Neutrophils (n $\times 10^{9} / \mathrm{l}$ ) & 14.2 & 3.7 & 8.2 & $1.5-8$ \\
\hline Platelets $\left(\mathrm{n} \times 10^{9} / \mathrm{L}\right)$ & 460 & 134 & 14 & $130-400$ \\
\hline C-reactive protein (mg/L) & - & 190. & 12 & $<5$ \\
\hline Creatinine (mg/dL) & 1.42 & 2.32 & 2.91 & $0.84-1.25$ \\
\hline
\end{tabular}


nosed with a JAK2-mutated MPN and he started cytoreduction with hydroxyurea with the aim to maintain platelets below $400 \times$ $10^{9} /$ L. Periodic follow-up was regularly performed and antiplatelet therapy continued.

In February 2018, the patient went into emergency department and was admitted to hospital because of persistent fever and worsening of renal function. Laboratory test showed a moderate normocytic anemia with increased inflammatory indexes and normal white blood cells (WBC) and platelet count (Table I). Blood cultures and Legionella and Pneumococcus urinary antigens were negative, while a chest X-ray revealed a left parenchymal thickening associated to pleural effusion. Broad spectrum antibiotic therapy with ceftriaxone and levofloxacine was started. Because of the worsening of renal function, an abdomen ultrasound (US) was performed and revealed left ureteronephrosis with presence of a suspected mass at the pyeloureteral joint. A concomitant splenomegaly was recorded with a spleen diameter of $22 \mathrm{~cm}$. The CT scan confirmed a hyperdense lesion which wrapped the left caliceal cavities (Figure 1). A similar finding, though less evident, was described at the right kidney. Imaging studies were repeated at the end of antibiotic therapy after resolution of fever and normalization of $\mathrm{C}$-reactive protein values, but the lesion appeared unmodified. In the following days, a rapid increase of $\mathrm{LDH}$ and the onset of leucocytosis with a $2 \%$ of CD $33^{+} \mathrm{CD} 34^{-}$

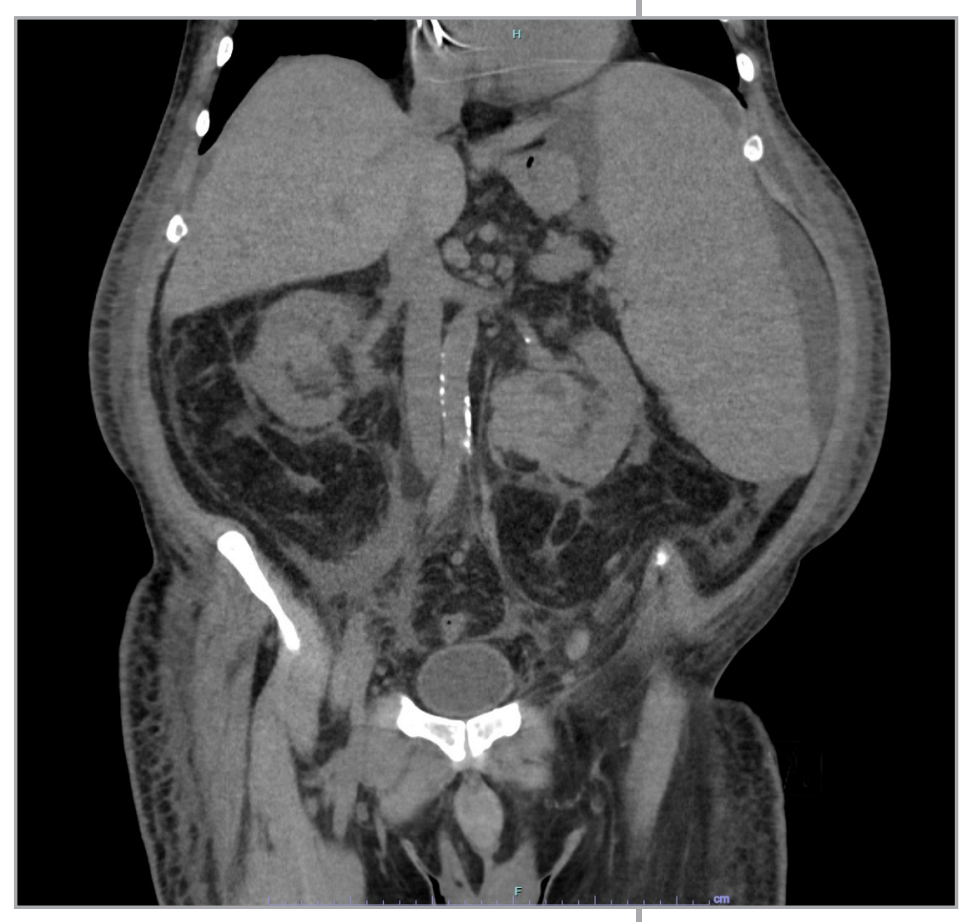

$\mathrm{CD}_{117^{+}} \mathrm{HLA}^{-D R}{ }^{+}$blasts in peripheral blood smear was observed. Therefore, a bone marrow evaluation and a US-guided needle biopsy of the left renal mass were performed. Both the histology profiles were consistent with acute monocytic leukemia (Figure 2).

At bone marrow aspiration, $25-30 \%$ of cellularity was made by blasts with the following immunophenotype: $\mathrm{CD} 45^{+/-} \mathrm{CD} 13^{+}$ $\mathrm{CD}_{3}{ }^{+} \mathrm{CD}_{36}{ }^{+} \mathrm{CD} 38^{+} \mathrm{CD} 117^{+} \mathrm{HLA}-\mathrm{DR}{ }^{+}$.

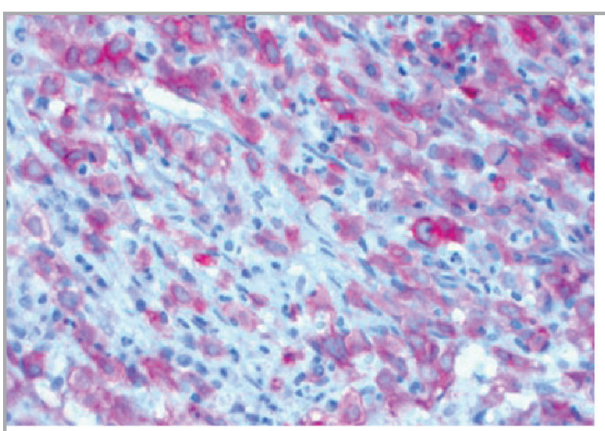

A

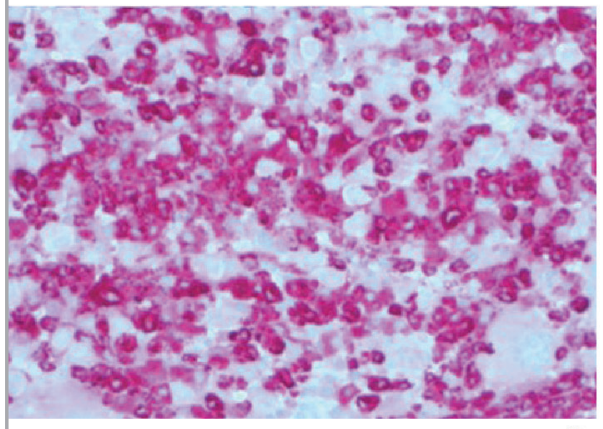

C
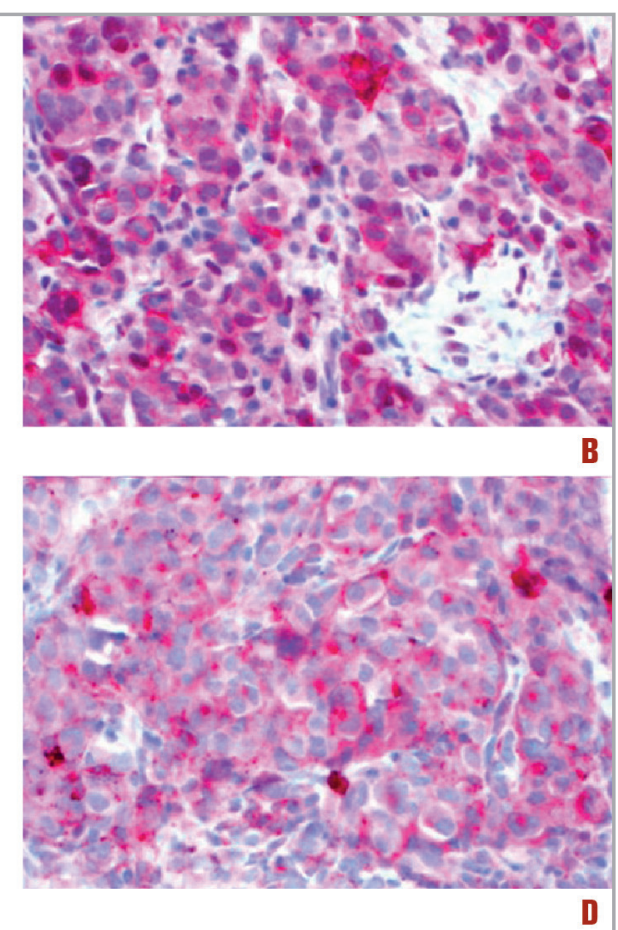

Figure 1. Abdominal computed tomography showing bilateral kidney solid lesions (more evident at the left kidney) together with the known splenomegaly.

Figure 2. Pelvic and bone marrow histology showing myeloid blastic cells. A) CD117 immunohistochemical analysis for blastsbone marrow biopsy, 40x; B) CD117 immunohistochemical analysis for blastspelvic mass biopsy, 40× C) lysozyme stainingbone marrow biopsy, 40x; D) lysozyme staining-pelvic mass biopsy, 40x 
Table II. Literature review of renal myeloid sarcoma.

$\mathrm{AML}=$ acute myeloid leukemia;

$\mathrm{CML}=$ chronic myeloid leukemia;

$\mathrm{CR}=$ complete remission $\mathrm{MS}=$ myeloid sarcoma

\begin{tabular}{|c|c|c|c|c|c|}
\hline & Diagnosis & $\begin{array}{l}\text { Leukemic } \\
\text { evolution }\end{array}$ & $\begin{array}{l}\text { Benal } \\
\text { function }\end{array}$ & Treatment & Outcome \\
\hline Park, 2003 [6] & $\begin{array}{l}\text { Non-leukemic } \\
\text { renal MS }\end{array}$ & No & Normal & $\begin{array}{c}\text { AML-like } \\
\text { chemotherapy }\end{array}$ & $\mathrm{CR}$ \\
\hline $\begin{array}{l}\text { Agrawal, } \\
2011 \text { [7] }\end{array}$ & Renal MS & $\begin{array}{l}\text { CML blast } \\
\text { crisis (mixed } \\
\text { phenotype) } \\
\text { after } 3 \text { months }\end{array}$ & Normal & None & $\begin{array}{l}\text { Lost to } \\
\text { follow-up }\end{array}$ \\
\hline $\begin{array}{l}\text { Palanisamy, } \\
\text { २०15 [8] }\end{array}$ & $\begin{array}{l}\text { Donor-derived } \\
\text { renal MS }\end{array}$ & No & Impaired & $\begin{array}{l}\text { Nephrectomy } \\
\text { + AML-like } \\
\text { chemotherapy }\end{array}$ & $\mathrm{CR}$ \\
\hline $\begin{array}{l}\text { Palanisamy, } \\
2015 \text { [8] }\end{array}$ & $\begin{array}{l}\text { Donor-derived } \\
\text { renal MS }\end{array}$ & No & Impaired & Nephrectomy & $\mathrm{CR}$ \\
\hline $\begin{array}{l}\text { Wang, } \\
2017 \text { [9] }\end{array}$ & $\begin{array}{l}\text { MS (intestine, } \\
\text { kidneys, } \\
\text { mesenteric } \\
\text { lymph nodes) }\end{array}$ & $\begin{array}{l}\text { AML after } 3 \\
\text { months }\end{array}$ & Not reported & $\begin{array}{c}\text { AML-like } \\
\text { chemotherapy }\end{array}$ & $\mathrm{CR}$ \\
\hline
\end{tabular}

As the patient was not eligible for intensive chemotherapy or surgery, treatment with low-dose cytarabine was started. However, general conditions rapidly worsened with the development of acute renal failure and subsequent death.

\section{DISCUSSION}

Myeloid sarcoma is a rare entity which may occur in the context of a myeloid neoplasia or rarely precede it. Presenting symptoms are varied and depend on the site of involvement, most frequently skin, bone, soft tissue, lymph nodes, reproductive or digestive organs, and central nervous system. To our knowledge, only few cases of renal MS have been described in literature and we summarized them in Table II [6-9]. Other sporadic cases were published in case series, but no detailed clinical information was provided.

In our case, the patient was previously affected by a JAK2-mutated chronic myeloproliferative neoplasm and the appearance of kidney MS was the first sign of leukemic evolution, which was overt only some weeks later. This presentation is in line with literature data, that showed that non-leukemic MS almost invariably progress to acute leukemia with a mean duration of 10 months or less. When MS occurs before the onset of overt leukemia, it is often misdiagnosed, especially when the involved site is not common, as in our case. Histologic approach is always needed to confirm a radiologic suspicion. MS radiologically appear as soft tissue masses on plain CT and as well-defined ho- mogeneously enhanced masses on enhanced CT. Differential diagnoses of renal MS on CT mainly include insignificantly enhanced renal cell carcinomas, renal infarctions, and renal lymphomas. Unlike renal MS, insignificantly enhanced renal cell carcinomas are usually singular and prone to hemorrhage, necrosis, and cystic changes in the lesions. Renal infarctions are usually wedge-shaped with no mass effect and enhanced cortical rim signs representative of cortical rims and renal vessel abnormalities on the lesion side may be observed on contrast-enhanced CT images. The CT characteristics of renal lymphomas include multiple lesions, which appear as masses with mild homogenous enhancement, and rarely with hemorrhage, necrosis, cystic changes, and calcification in the lesions. These characteristics complicate the differential diagnosis of renal MS.

When MS occurs as a presenting feature before the onset of overt leukemia, it is often misdiagnosed as lymphoma. A review of 72 patients of non-leukemic MS showed that 35 patients $(47 \%)$ were initially misdiagnosed, most often (31/35) as malignant lymphoma. The morphological similarity of blasts with lymphoma cells, especially in the blastic and undifferentiated variant of MS and its rarity, resulting in low index of suspicion, have been reported as the main reasons for the misdiagnosis in such cases. The majority of extramedullary blastic tumors can be classified as pre $\mathrm{B}$, pre $\mathrm{T}$, or MS using a panel of monoclonal antibodies including myeloid (MPO, lysozyme, CD68, and CD43), together with $\mathrm{B}$ lineage markers (CD20 and CD79a) and T lineage markers 
(45RO and CD3) [10]. Therefore, correct pathological diagnosis at the right time is a must and requires the pathologist to maintain a high index of suspicion of MS when viewing a lesion that resembles large cell lymphoma, carrying out special studies with a complete panel of antibodies.

\section{CONCLUSION}

In conclusion, our case highlights the possibility of kidney involvement by MS before leukemic manifestation of disease. The radiological examination shows kidney lesions as soft tissue masses, which generally present a homogenous enhancement. MS should be considered in the differential diagnosis, and an aspiration biopsy should be performed to provide a definitive pathological diagnosis. If MS is diagnosed, systemic chemotherapy is crucial to recovery, otherwise the disease may progress rapidly. Imaging is helpful in the differential diagnosis of MS, as well as in evaluating and monitoring the response of patients to treatment.

\section{Keypoints}

- In patients with a history of myeloid neoplasm, even in the absence of other evidence of active disease, MS should be considered in the differential diagnosis of collecting system tumors.

- Histologic confirmation of the nature of a renal mass is needed in order to start the appropriate treatment

- Although rare, early and accurate multidisciplinary diagnosis is important in order to start treatment of MS as soon as possible to increase survival rate

- Further studies are needed to investigate the clinical features and pathogenesis of this condition

\section{Consent for publication}

The consent for publication was obtained from a relative of the patient here described.

\section{Funding}

This article has been published without the support of sponsors.

\section{Conflicts of interests}

The authors declare they have no competing financial interests concerning the topics of this article.

\section{REFERENCES}

1. Arber DA, Orazi A, Hasserjian R, et al. The 2016 revision to the World Health Organization classification of myeloid neoplasms and acute leukemia. Blood 2016; 127: 2391-405; https:// doi.org/10.1182/blood-2016-03-643544

2. Wang HQ, Li J. Clinicopathological features of myeloid sarcoma: Report of 39 cases and literature review. Pathol Res Pract 2016;212: 817-24; https://doi.org/10.1016/j.prp.2016.06.014

3. Al-Khateeb H, Badheeb A, Haddad H, et al. Myeloid sarcoma: clinicopathologic, cytogenetic, and outcome analysis of 21 adult patients. Leuk Res Treat 2011; 2011: 523168; https://doi. org $/ 10.4061 / 2011 / 523168$

4. Bakst RL, Tallman MS, Douer D, et al. How I treat extramedullary acute myeloid leukemia. Blood 2011; 118: 3785-93; https://doi.org/10.1182/blood-2011-04-347229

5. Li JM, Liu WP, Zhang MH, et al. Chen Clinicopathologic and immunophenotypic analysis of myeloid sarcoma. Zhonghua Bing Li Xue Za Zhi 2006; 35: 606-11

6. Park HJ, Jeong DH, Song HG, et al. Myeloid sarcoma of both kidneys, the brain, and multiple bones in a nonleukemic child. Yonsei Med J2003; 44: 740-3; https://doi.org/10.3349/ ymj.2003.44.4.740 
7. Agrawal V, Gupta A, Gupta R, et al. Nonleukemic granulocytic sarcoma of kidney with mixed phenotype blasts: a diagnostic dilemma. Indian J Pathol Microbiol 2011; 54: 606-8; https://doi. org/10.4103/0377-4929.85111

8. Palanisamy A, Persad P, Koty PP, et al. Donor-derived myeloid sarcoma in two kidney transplant recipients from a single donor. Case Rep Nephrol 2015; 2015: 821346; https://doi. org/10.1155/2015/821346

9. Wang P, Li Q, Zhang L, et al. A myeloid sarcoma involving the small intestine, kidneys, mesentery, and mesenteric lymph nodes: A case report and literature review. Medicine (Baltimore) 2017; 96: e7934; https://doi.org/10.1097/MD.0000000000007934

10. Traweek ST, Arber DA, Rappaport H. Extramedullary myeloid cell tumors: An immunohistochemical and morphologic study of 28 cases. Am J Surg Pathol 1993; 17: 1011-9; https://doi.org/10.1097/00000478-199310000-00006 